(2)

\title{
Mesenteric cryptococcal granuloma in a dog caused by Cryptococcus neoformans var. grubii
}

This article was published in the following Dove Press journal:

Veterinary Medicine: Research and Reports

27 September 2012

Number of times this article has been viewed

Jennifer R Cook

Karen E Russell

Kristin B Eden

Aline Rodrigues-Hoffmann

Department of Veterinary

Pathobiology, College of Veterinary

Medicine and Biomedical Sciences,

Texas A\&M University, College Station,

TX, USA
Correspondence: Jennifer R Cook CVM-TAMU-Clin Path; Building 1085 ,

Room 2020; College Station,

TX 77843, USA

Tel + I 9798459180

$\mathrm{Fax}+\mid 979458$ । 325

Email jcook@cvm.tamu.edu

\begin{abstract}
Although cryptococcosis is usually associated with respiratory and neurologic signs in domestic species (such as sneeze, cough, nasal discharge, seizures, ataxia), clinical manifestations of the disease may be more subtle and nonspecific. A 3-year-old male castrated Boxer dog presented with a history of chronic vomiting, diarrhea, weight loss, and lethargy. At no time had respiratory or neurologic signs been noted by the owners or the primary care veterinarian. Palpation of an abdominal mass revealed an atypical lesion location: a large $(16 \times 9 \times 7 \mathrm{~cm})$ mass at the root of the mesentery. Diagnosis was achieved through cytology of this mass and a positive serologic Cryptococcus capsular antigen titer; polymerase chain reaction was utilized for speciation of the abdominal isolate as Cryptococcus neoformans variety grubii. The animal was euthanized due to poor prognosis. After necropsy and histopathologic analysis, the mesenteric mass and associated lymph nodes were identified as large fungal granulomas. This is a rare manifestation of cryptococcosis, involving several visceral organs, with no remaining evidence of the route of entry of the organism. As prompt diagnosis of mycotic illness is paramount to successful management, this case indicates that cryptococcal infection should be considered as a differential diagnosis in dogs with gastrointestinal signs and lymphadenopathy. The protean nature of cryptococcosis is discussed within the context of a brief review of emerging and unresolved issues in pathogenesis.
\end{abstract}

Keywords: Cryptococcus gattii, granuloma, lymphadenitis

\section{Introduction}

There are over thirty species within the Cryptococcus genus; however, to date only two have proved to be medically important pathogens: $C$. neoformans and $C$. gattii. In humans and domestic animals, infection is typically initiated via respiratory exposure, but the organism may also be ingested or implanted directly into cutaneous wounds or into the teat canal. ${ }^{1,2}$ If exposure and infection progress to clinical illness, the most common presenting signs are respiratory and neurologic/ocular, reflecting the major route of infection and the organism's predilection for the central nervous system. ${ }^{1-5}$ The young Boxer dog presented here had no history of respiratory or neurologic signs throughout a 3-month illness; thus, cryptococcal infection was not an initial diagnostic consideration. This case report highlights an atypical clinical and pathologic presentation in a case of canine cryptococcosis with involvement of the abdominal viscera.

\section{Case report}

A 3-year-old male castrated Boxer dog presented to the Veterinary Medical Teaching Hospital at Texas A\&M University because of a 3-month history of weight loss 
(approximately 16 kilograms), vomiting (twice weekly), and diarrhea (once weekly) that had been unresponsive to symptomatic therapy. The animal was not on any medications except monthly ivermectin used for heartworm preventation. A mid-abdominal mass had been palpated by the referring veterinarian, of which radiographs had been inconclusive. At presentation, the dog weighed $25.2 \mathrm{~kg}$ and was in slightly thin body condition (score 3/9; prior obesity was reported). Body temperature, heart rate, respiratory rate, and pulse quality were unremarkable. Clinicopathologic data included the following abnormalities: mild leukocytosis $(18,000$ leukocytes/ $\mu \mathrm{L}$; reference interval $6000-17,000 / \mu \mathrm{L}$ ) characterized by mature neutrophilia $(15,840$ neutrophils/ $\mu \mathrm{L}$; reference interval 3000-11,500/ $\mu \mathrm{L})$; mild hyperproteinemia ( $8.3 \mathrm{~g} / \mathrm{dL}$; reference interval $6.0-8.0 \mathrm{~g} / \mathrm{dL})$; moderate hyperglobulinemia $(6.0 \mathrm{~g} / \mathrm{dL}$; reference interval $1.7-3.8 \mathrm{~g} / \mathrm{dL})$; mild hypernatremia (148 mmol/L; reference interval 139-147 mmol/L); mild hyperchloremia (119 $\mathrm{mmol} / \mathrm{L}$; reference interval 107-116 mmol/L); low alanine aminotransferase activity ([ALT] < $6 \mathrm{U} / \mathrm{L}$; reference interval 10-130 U/L); poor urine concentration (urine-specific gravity 1.014); and slightly elevated urinary $\mathrm{pH}$ (7.5; reference interval 6.0-7.0) with inactive urinary sediment.

A firm cranioabdominal mass was palpated, and abdominal ultrasound revealed a $9.6 \times 7.1 \mathrm{~cm}$ complex hypoechoic mass involving the caudate liver lobe and ileum. Medial iliac and abdominal lymph nodes were enlarged, and there was a small amount of peritoneal fluid present. A sample from the mass was aspirated and submitted for cytologic analysis. Cytologic smears were highly cellular, with moderate to marked pyogranulomatous inflammation and numerous extracellular and intrahistiocytic magenta to occasionally light bluegreen yeast organisms, approximately 5-20 $\mu \mathrm{m}$ in diameter, with prominent nonstaining capsules and $1 \mu \mathrm{m}$ diameter walls that were occasionally folded, giving a triangular or comma-shaped appearance overlaying the center of the organism (Figure 1). Yeast occasionally displayed narrowbased budding, infrequently producing chains of three to five organisms. Based on these morphologic characteristics, a presumptive diagnosis of visceral cryptococcosis was made. A cryptococcal capsular polysaccharide antigen latex agglutination titer was positive at 1:1024. Due to the severity of the dog's clinical signs and the extent of the abdominal lesion with poor associated prognosis, euthanasia was elected.

At necropsy, a $16 \times 9 \times 7 \mathrm{~cm}$ firm, irregular, multinodular, tan to red mass was adhered to the root of the mesentery (Figure 2). The mass was observed to compress, but not

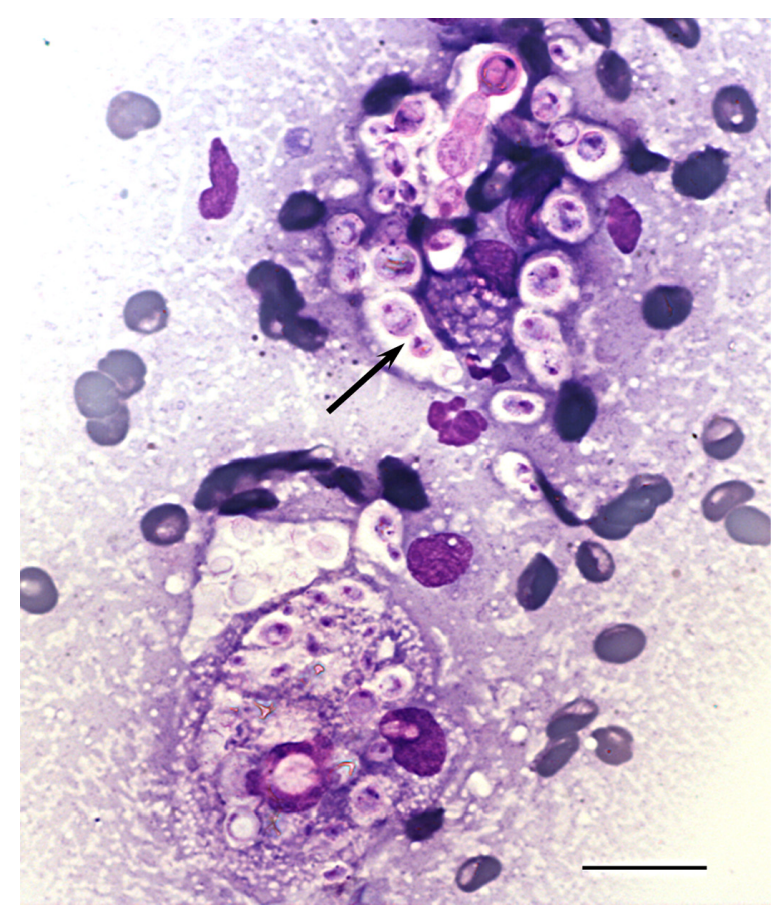

Figure I Fine-needle aspirate cytology of a cranial abdominal mass displaying numerous extracellular and intrahistiocytic Cryptococcus spp. yeasts with prominent nonstaining capsules, narrow-based budding (arrow), and occasional chains of organisms. Notes: Modified Wright's stain (Diff-Quik ${ }^{\circledR}$ ). $60 \times$ objective. Bar $=20 \mu \mathrm{m}$.

infiltrate, the head of the pancreas, the caudate liver lobe, and the intestinal wall at the duodenal-jejunal and ileocecocolic junctions, resulting in narrowing of the intestinal lumen. On cut section, the mass contained multifocal cavitated, yellow, friable areas of necrosis. Additionally, cranial mediastinal, tracheobronchial, and inguinal lymph nodes were enlarged, nodular, firm, and mottled tan to red and white. Histologically, the mesenteric mass was composed of severe granulomatous inflammation characterized by large numbers of epithelioid

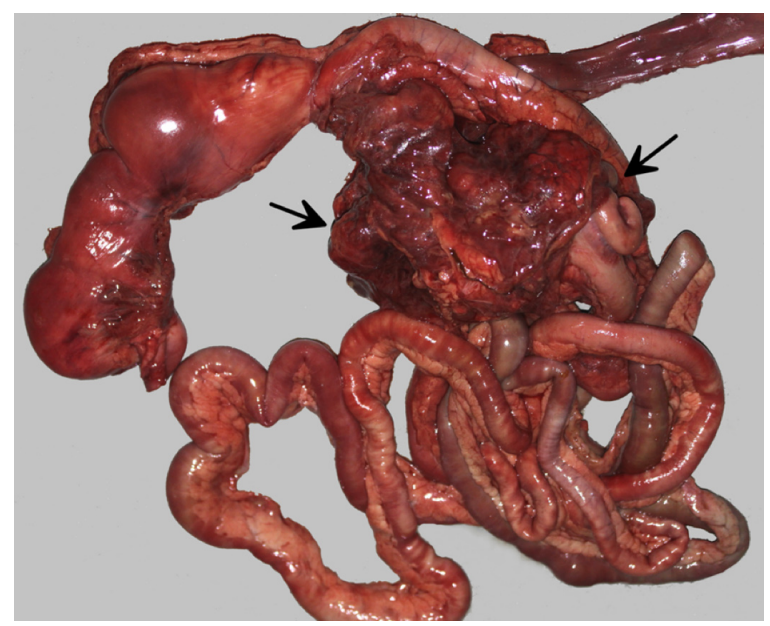

Figure 2 Gross necropsy photograph of intestinal tract with large cryptococcal lesion (demarcated by arrows) adhered to the root of the mesentery. 
macrophages, lymphocytes, plasma cells, and numerous multinucleated giant cells, with multifocal areas of necrosis, edema, and abundant fibrin deposition. Admixed within inflammatory cells were large numbers of 5-15 $\mu \mathrm{m}$ diameter, round, amphophilic, refractile, extracellular and occasionally intracellular fungal yeasts with 3-5 $\mu \mathrm{m}$ diameter clear capsules, which stained bright pink-red with mucicarmine stain (Figure 3).

Mesenteric and mediastinal lymph nodes were largely effaced by similar inflammation. Mesenteric nodes had abundant fibrous connective tissue that extended into the serosa of the associated small intestine but not into the tunica muscularis, submucosa, or mucosa. Rare organisms were also observed in the center of a single granuloma within the renal pelvis and focally within the red pulp of the spleen. The heart and liver contained mild multifocal lymphoplasmacytic and histiocytic inflammation, but no organisms were observed in these tissues. No organisms or significant inflammation were observed in the nasal turbinates, lungs, or gastrointestinal tract. DNA extracted from a formalinfixed, paraffin-embedded section of the granuloma was used as a template in a polymerase chain reaction (PCR) using panfungal internal transcribed spacer primers ITS4 (5'-TCC TCC GCT TAT TGA TAT GC-3') and ITS5 (5'-GGA AGT AAA AGT CGT AAC AAG G-3') to amplify the ITS region (ITS1-5.8S-ITS-2) of the ribosomal RNA gene as previously described. ${ }^{6}$ Sequences were confirmed by sequencing both strands in duplicate, and forward and reverse sequences were aligned by a sequence alignment program. ${ }^{7}$ The sequence from the granuloma tissue most closely matched that of Cryptococcus neoformans var. grubii (teleomorph Filobasidiella neoformans serotype A) with 100\% sequence

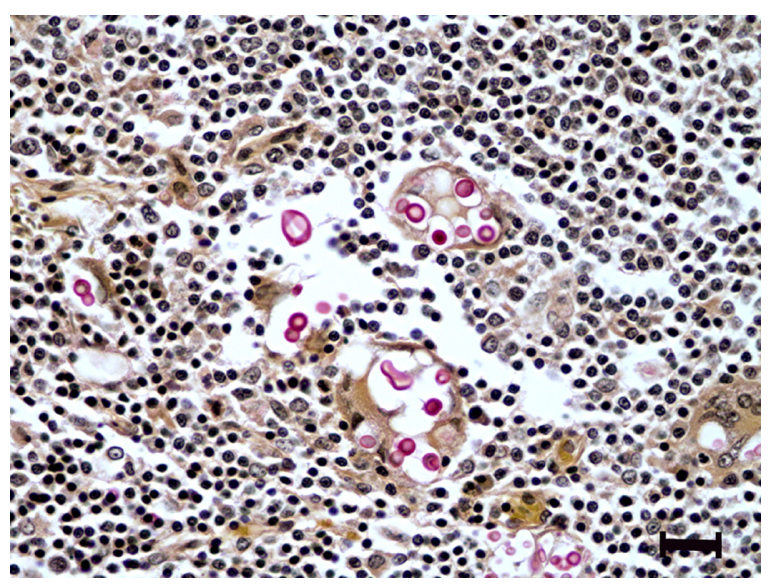

Figure 3 Histologic section of mesenteric lymph node with histiocytic inflammation and extracellular cryptococcal yeast organisms (pink).

Notes: Mucicarmine stain. $\mathrm{Bar}=20 \mu \mathrm{m}$. identity to GenBank ${ }^{\circledR}$ accession \#AJ876598 and to CBSKNAW Fungal Biodiversity Centre database (Utrecht, The Netherlands) accession \#8710.

\section{Discussion}

Since the first documented human infection in 1894, there has been increasing awareness, reporting, and study of cryptococcosis in domestic species. ${ }^{8}$ Scientific inquiry has been accelerated due to several recent developments. Firstly, the global increase in the number of immunosuppressed people due to HIV, advances in cancer chemotherapy, and increased frequency of organ transplantation has witnessed a concomitant increase in the number of human cases of clinical fungal infection. ${ }^{9,10}$ Secondly, recent reclassification of the former $C$. neoformans var. gattii into its own species, C. gattii (serotypes B and C), and the subclassification of $C$. neoformans into two varieties, C. neoformans var. neoformans, (serotype D and hybrid serotype AD), and C. neoformans var. grubii (serotype A), has raised issues of fungal genetic selection, differential virulence, and molecular pathogenesis. ${ }^{9-11}$ Lastly, the emergence of a new geographic locus of infection of $C$. gattii with increased human and animal cases, beginning on Vancouver Island in 1999 (and currently involving Vancouver, British Columbia, Washington, Idaho, Oregon, and California), has challenged the traditional understanding of the ecologic range of the organism and the ability of seemingly immunocompetent hosts to successfully combat exposure and subclinical infection. ${ }^{11-13}$

There is mounting evidence that clinical disease is the least common outcome of exposure to Cryptococcus spp., suggesting that the immune status of the host plays a crucial role. Antibody testing, although not useful for diagnosis of clinical disease, may shed light on the prevalence of exposure, subclinical infection, or prior infection. Limited studies of prevalence of anticapsular antibodies among healthy persons suggest significant exposure (up to $98 \%$ seroprevalence of immunoglobulin $\mathrm{M}$ and up to $70 \%$ immunoglobulin G), assuming that the measured antibodies are not simply cross-reactive to different microorganisms. ${ }^{14,15}$ One study has shown a lower prevalence (3\%-10\%) of asymptomatic, antibody-positive, cats, dogs, horses, and koalas ( $\mathrm{n}=314$; only koalas had tested as definitively antigen-negative). ${ }^{16}$ Necropsy findings in humans and animals, as well as culture, antigen serology, and DNA analysis of clinical isolates, have demonstrated the presence of subclinical infections with potentially protracted dormancy. ${ }^{17,18}$ 
As is the case for many fungal infections, host response mainly involves innate and $\mathrm{T}$ lymphocyte helper 1/T lymphocyte helper 17-driven cell-mediated immunity, with phagocyte killing largely promoted by interferon gamma. However, the organism can survive and replicate within phagocytes (predominantly macrophages), and the rate of replication has been directly linked to virulence in a murine model. ${ }^{19}$ The role of mononuclear cells in latency and dissemination has been further supported by research demonstrating that yeasts may be transferred directly between host cells, extricate themselves from phagocytes without killing the host cell (without inciting inflammation), and be passed directly from phagocytes to endothelial cells when crossing the blood-brain barrier (the "Trojan Horse" theory of dissemination). ${ }^{20}$ These recent and ongoing research efforts provide new understanding of the molecular pathogenesis behind the potentially silent subclinical spread of the organism to sites remote from initial infection.

Case series of domestic species (including dogs, cats, horses, alpacas, ferrets, sheep, and birds) have documented up to a $45 \%$ rate of infection at atypical sites (with or without common site involvement), including viscera, heart, oral cavity/salivary gland, thyroid gland, mediastinum, and bone/ joints. ${ }^{1-3,5}$ In regard to reported cases in canines and felines (the most represented veterinary species), cats are more frequently infected; however, dogs have a higher proportion of disseminated disease to the central nervous system and to atypical sites. ${ }^{1,3,5}$ Full necropsy data from many of these cases of cryptococcosis are lacking, precluding definitive evaluation of the initial site of infection and the full extent of lesions. To the authors' knowledge, visceral cryptococcosis, distinctly reported without respiratory, neurologic/ocular, or cutaneous involvement, has been specifically highlighted in the literature in four canines, a koala, a ferret, and a horse. ${ }^{2,4,21-25}$ These reports also noted nonspecific gastrointestinal signs and weight loss among patients. Four larger veterinary case series document a low to moderate incidence of atypical sites but do not specify whether these cases also involved more common sites. ${ }^{1-3,5}$ In a recent study of $31 \mathrm{dogs}$ with varied presentations of cryptococcosis, $16 \%$ had vomiting as part of the clinical presentation. ${ }^{3}$ In the dog of the present report, it is likely that vomiting and diarrhea occurred secondarily to mechanical ileus imposed by the adherent mesenteric granuloma.

More than half of the few case reports of visceral disease in domestic species display infiltration into the wall of the intestinal tract. ${ }^{21-25}$ Regarding visceral cases without gastrointestinal lesions, ingestion and gastrointestinal dissemination may be followed by local clearance of organisms or failure to detect the original gastrointestinal lesion(s). Alternatively, visceral and/or enteric structures may become affected after primary respiratory or cutaneous infection and subsequent vascular dissemination. Interestingly, older studies involving experimental feeding of organisms to hamsters and monkeys resulted in lesions in the mesenteric lymph nodes, liver, and lung, but absence of detectable gastrointestinal lesions. ${ }^{26,27}$ For the dog of this case, the absence of gross cutaneous/gross and histologic respiratory and gastrointestinal lesions resulted in the inability to document the original route of entry. This may be a reflection of the chronicity of the course of disease, highlighting the ability of Cryptococcus spp. to successfully migrate remotely through the vasculature, obscuring diagnosis in a setting of protracted disease. It is also possible that lack of tissue culture and the postmortem histologic sampling of a limited number of presumptively representative sites may have led to a false negative documentation of the route of primary infection.

It is important to note that, as in this dog, visceral lymphadenitis (typically featuring thoracic, mesenteric, iliac, and/or inguinal lymph nodes) is a frequent feature of disseminated cryptococcosis (up to $35.5 \%$ of canine cases in one study), regardless of the presenting signs or site(s) of organ involvement, giving further support to the importance of vascular/lymphatic dissemination via mononuclear cells. ${ }^{3,22,24,25}$ Thus, lymphadenopathy alone should prompt cryptococcal infection as a differential diagnosis. Of note, in a case review of necropsied horses with cryptococcosis, mesenteric lymph node involvement occurred in all cases displaying diffuse miliary lung lesions and not in any cases with larger caudodorsal pulmonary granulomas, suggesting differing modes of dissemination (vascular vs inhalation, respectively) to the lung fields. ${ }^{1}$

The dog of this case was 3 years old. Published data of canine cryptococcosis of any manifestation indicates a significant overrepresentation of young (less than 5 years) animals. ${ }^{1-}$ ${ }_{5,22,23}$ A similar age distribution has been documented in a limited number of equine cases, but there is a more random distribution in cases involving felines and koalas. . $^{1,24,25}$ In humans, age distribution is symmetric or skewed toward older patients, presumably due to development of comorbid immunosuppressive conditions. ${ }^{28}$ The differential age phenomenon in canine cryptococcosis has been theoretically attributed to issues of exposure, with young dogs presumptively spending more time outdoors in contact with plant matter or bird/bat excrement, which are favorable growth media for 
the organism. The dog of this report spent approximately 3-4 hours per day outdoors in a fenced yard that was free of lush vegetation or bird/bat feces, and he occasionally swam in a pond on the property. There were no pet birds on the property. Alternatively, the young age of dogs with Cryptococcus spp. infections has been attributed to species-specific immune responses, with felines, humans, and koalas better able to maintain the organism in a subclinical state. ${ }^{1}$ The pattern warrants further study, including the role of initial load of infectious particles on the balance between clearance, subclinical infection, and clinical disease.

C. neoformans var. grubii, the variant isolated in this case, is responsible for $95 \%$ of worldwide cases of human cryptococcosis, with approximately one percent caused by C. gattii. $^{19}$ No such statistics exist for domestic species; pooled data from five large published veterinary clinical case series $(n=20-195$; primarily felines and canines, with twenty horses, three alpacas, four ferrets, one sheep, and one bird) reveal a known species prevalence of $62.5 \%$ (167/267) for $C$. neoformans and 37.5\% (100/267) for C. gattii. $^{1-5}$ These data are limited in that few veterinary clinical isolates are speciated, and also because these cases occurred in C. gattii-endemic areas (Australia, California, and western Canada). There is no known correlation between infecting species and clinical signs, extent of lesions, or response to therapy. ${ }^{3,5}$ Speciation of cryptococcal organisms can be performed via culture or PCR, and remains important from an epidemiologic standpoint of surveillance for the emergence/ spread of $C$. gattii within novel environments ( $C$. neoformans is ubiquitous), and for the detection of potential future strains of enhanced virulence.

The dog of this report was previously healthy, presumed to be immunocompetent, and lived with two other unaffected dogs (negative Cryptococcal antigen tests), including another young Boxer with no genetic relationship. Other animals on the property (ten horses, one steer, three barn cats, chickens, and two pigs) have also remained clear of clinical disease. In humans, cryptococcosis has traditionally been understood as a disease of immunosuppression. Statistics are varied (19\%-62\%) regarding the prevalence of clinical cases among immunocompetent humans. ${ }^{28}$ The outbreak of C. gattii in the North American Pacific Northwest has been noteworthy due to reports of increased numbers of human cases among immunocompetent individuals, leading to the conclusion that $C$. gattii is more virulent than $C$. neoformans. ${ }^{11}$ The issue is controversial and may be confounded by differences in microorganism prevalence and by differential exposure between immunocompetent and immunosuppressed individuals. All known major phenotypic virulence factors are shared between $C$. neoformans and C. gattii, and studies of genetic and proteomic mechanisms of differential virulence between the two species, and between different strains of each species, are ongoing. Examples of new developments include differences in ability to proliferate within macrophages, ability to suppress neutrophil function, regulation of common transcription factors, and shared protein function. ${ }^{11,19}$

The issue of immunocompetence is difficult for veterinarians to assess without documentation of an underlying disease or prior administration of immunosuppressive medications. A great majority of domestic and wild animals with cryptococcosis have no known history of immunosuppression. One study from the Pacific Northwest documented $21 \%$ of 78 clinically infected cats and $12 \%$ of 51 clinically infected dogs to have an underlying, potentially immunosuppressive disease. This study showed no correlation between comorbidity and mortality. ${ }^{29}$ Although there have been differing reports on the significance of coinfection with Cryptococcus spp. and either feline leukemia or feline immunodeficiency viruses, recent research has not shown cats with retroviral infections to be overrepresented in cases of feline cryptococcosis, nor does coinfection appear to affect treatment outcome. ${ }^{1,3,5}$ The lower reported proportions of domestic animals with immunosuppression and cryptococcosis, in comparison with humans, raises questions of decreased detection of immunosuppression in veterinary species, species/breed-specific immunity, differential exposure, and the role of infective load of organisms.

\section{Conclusion}

Prompt diagnosis of systemic infections is crucial to successful clinical management. Although still primarily a respiratory and neurologic pathogen statistically, an increasing body of case material has proven that cryptococcosis displays quite variable clinical presentations and progressions. The case presented here illustrates the need for heightened awareness among clinicians of the possibility of atypical, visceral cryptococcosis in dogs, especially young dogs, presenting with nonspecific signs (fever, anorexia, weight loss, lethargy), gastrointestinal signs (vomiting, diarrhea), and/or visceral lymphadenopathy. Fine needle aspirate cytology of enlarged visceral organs or mass lesions is a rapid and relatively noninvasive means of achieving a presumptive diagnosis while pending confirmatory testing, such as capsular antigen serology, fungal culture, histopathology, or PCR. As neurologic involvement has been shown to be 
the only independent predictor of mortality in dogs and cats, early detection with aggressive treatment, including possible surgical debulking of abdominal masses along with conventional antimicrobial drugs, will hopefully lead to a higher percentage of favorable outcomes. ${ }^{29}$

\section{Acknowledgments}

Mr Dan Bradway and the Molecular Diagnostics Laboratory at the Washington Animal Disease Diagnostic Lab performed $\mathrm{PCR} /$ sequencing and assisted with the technical portion of the text. Dr Wayne Corapi, Department of Pathobiology, Texas A\&M College of Veterinary Medicine and Biomedical Sciences, assisted with image preparation.

\section{Disclosure}

The authors have received no financial support for the research, authorship, and/or publication of this article and declare no potential conflicts of interest.

\section{References}

1. McGill S, Malik R, Saul N, et al. Cryptococcosis in domestic animals in Western Australia: a retrospective study from 1995-2006. Med Mycol. 2009;47(6):625-639.

2. Lester SJ, Kowalewich NJ, Bartlett KH, Krockenberger MB, Fairfax TM, Malik R. Clinicopathologic features of an unusual outbreak of cryptococcosis in dogs, cats, ferrets, and a bird: 38 cases (Jan-Jul 2003). $J$ Am Vet Med Assoc. 2004;225(11):1716-1722.

3. Trivedi SR, Sykes JE, Cannon MS, et al. Clinical features and epidemiology of cryptococcosis in cats and dogs in California: 93 cases (1988-2010). J Am Vet Med. Assoc. 2011;239(3):357-369.

4. Malik R, Dill-Macky E, Martin P, Wigney DI, Muir DB, Love DN. Cryptococcosis in dogs: a retrospective study of 20 consecutive cases. J Med Vet Mycol. 1995;33(5):291-297.

5. O'Brien CR, Krockenberger MB, Wigney DI, Martin P, Malik R. Retrospective study of feline and canine cryptococcosis in Australia from 1981 to 2001: 195 cases. Med Mycol. 2004;42(5):449-460.

6. Katsu M, Kidd S, Ando A, et al. The internal transcribed spacers and 5. $8 \mathrm{~S}$ rRNA gene show extensive diversity among isolates of the Cryptococcus neoformans species complex. FEMS Yeast Res. 2004; 4(4-5):377-388.

7. Thompson JD, Higgins DG, Gibson TJ. CLUSTAL W: improving the sensitivity of progressive multiple sequence alignment through sequence weighting, position-specific gap penalties and weight matrix choice. Nucleic Acids Res. 1994;22(22):4673-4680.

8. Busse O. Ueber parasitäre Zelleninschlüsse und ihre Züchtung. [On parasitic cell inclusions and their cultivation]. Centralbl. f. Bakter, I. Abt. Orig. Bakterial. 1894;16:175-180. German.

9. Chayakulkeeree M, Perfect JR. Cryptococcosis. Infect Dis Clin North Am. 2006;20(3):507-544.

Veterinary Medicine: Research and Reports

\section{Publish your work in this journal}

Veterinary Medicine: Research and Reports is an international, peer-reviewed, open access journal publishing original research, case reports, editorials, reviews and commentaries on all areas of veterinary medicine. The manuscript management system is completely online and includes a very quick and fair peer-review system.
10. Lin X. Cryptococcus neoformans: morphogenesis, infection, and evolution. Infect Genet Evol. 2009;9(4):401-416.

11. Chaturvedi V, Chaturvedi S. Cryptococcus gattii: a resurgent fungal pathogen. Trends Microbiol. 2011;19(11):564-571.

12. Stephen C, Lester S, Black W, Fyfe M, Raverty S. Multispecies outbreak of cryptococcosis on southern Vancouver Island, British Columbia. Can Vet J. 2002;43(10):792-794.

13. Byrnes EJ III, Li W, Lewit Y, et al. Emergence and pathogenicity of highly virulent Cryptococccus gattii genotypes in the northwest United States. PLoS Pathog. 2010;6(4):e1000850.

14. Goldman DL, Khine H, Abadi J, et al. Serologic evidence for Cryptococcus neoformans infection in early childhood. Pediatrics. 2001;107(5):e66.

15. Houpt DC, Pfrommer GS, Young BJ, Larson TA, Kozel TR. Occurrences, immunoglobulin classes, and biological activities of antibodies in normal human serum that are reactive with Cryptococcus neoformans glucuronoxylomannan. Infect Immun. 1994;62(7):2857-2864.

16. Malik R, Speed BR, Kaldor J, et al. Serum antibody response to Cryptococcus neoformans in cats, dogs and koalas with and without active infection. Med Mycol. 1999;37(1):43-51.

17. Krockenberger MB, Canfield PJ, Barnes J, et al. Cryptococcus neoformans var. gattii in the koala (Phascolarctos cinereus): serologic evidence for subclinical cryptococcosis. Med Mycol. 2002;40(3):273-282.

18. Duncan C, Stephen C, Lester S, Bartlett, KH. Sub-clinical infection and asymptomatic carriage of Cryptococcus gattii in dogs and cats during an outbreak of cryptococcosis. Med Mycol. 2005;43(6):511-516.

19. Ma H, Hagen F, Stekel DJ, et al. The fatal fungal outbreak on Vancouver Island is characterized by enhanced intracellular parasitism driven by mitochondrial regulation. Proc Natl Acad Sci U S A. 2009;106(31): 12980-12985.

20. Charlier C, Nielsen K, Daou S, Brigitte M, Chretien F, Dromer F. Evidence of a role for monocytes in dissemination and brain invasion by Cryptococcus neoformans. Infect Immun. 2009;77(1):120-127.

21. Graves TK, Barger AM, Adams B, Krockenberger MB. Diagnosis of systemic cryptococcosis by fecal cytology in a dog. Vet Clin Pathol. 2005;34(4):409-412.

22. Malik R, Hunt GB, Bellinger CR, et al. Intra-abdominal cryptococcosis in two dogs. J Small Anim Pract. 1999;40(8):387-391.

23. van der Gaag I, van Niel MH, Belshaw BE, Wolvekamp WT. Gastric granulomatous cryptococcus mimicking gastric carcinoma in a dog. Vet $Q .1991 ; 13(4): 185-190$.

24. Krockenberger MB, Canfield PJ, Malik R. Cryptocococcus neoformans var. gattii in the koala (Phascolarctos cinereus): a review of 43 cases of cryptococcosis. Med Mycol. 2003;41(3):225-234.

25. Riley CB, Bolton JR, Mills JN, Thomas JB. Cryptococcosis in seven horses. Aust Vet J. 1992;69(6):135-139.

26. Sethi KK. Attempts to produce experimental intestinal cryptococcosis and sporotrichosis. Mycopathol Mycol Appl. 1967;31(3):245-250.

27. Takos MJ. Experimental cryptococcosis produced by the ingestion of virulent organisms. N Engl J Med. 1956;254(13):598-601.

28. Centers for Disease Control and Prevention. Emergence of Cryptococcus gattii - Pacific Northwest, 2004-2010. MMWR Morb and Mortal Wkly Rep. 2010;59(28):865-868.

29. Duncan C, Stephen C, Campbell J. Clinical characteristics and predictors of mortality for Cryptococcus gattii infection in dogs and cats of southwestern British Columbia. Can Vet J. 2006;47(10):993-998.

\section{Dovepress}

Visit http://www.dovepress.com/testimonials.php to read real quotes from published authors. 